\title{
AN INTRINSIC BALDWIN EFFECT IN THE H $\beta$ BROAD EMISSION LINE IN THE SPECTRUM OF NGC 5548
}

\author{
Karoline M. Gilbert ${ }^{1}$ and Bradley M. Peterson \\ Department of Astronomy, Ohio State University, 140 West 18th Avenue, Columbus, OH 43210-1173; \\ kgilbert@astro.ucsc.edu, peterson@astronomy.ohio-state.edu \\ Received 2002 August 27; accepted 2002 December 16
}

\begin{abstract}
We investigate the possibility of an intrinsic Baldwin effect (i.e., nonlinear emission-line response to continuum variations) in the broad $\mathrm{H} \beta$ emission line of the active galaxy NGC 5548 using cross-correlation techniques to remove light-travel time effects from the data. We find a nonlinear relationship between the $\mathrm{H} \beta$ emission line and continuum fluxes that is in good agreement with theoretical predictions. We suggest that similar analysis of multiple lines might provide a useful diagnostic of physical conditions in the broad-line region.
\end{abstract}

Subject headings: galaxies: active — galaxies: individual (NGC 5548) — galaxies: nuclei — galaxies: Seyfert

\section{INTRODUCTION}

Correlations between the continuum and emission-line properties of active galactic nuclei (AGNs) afford potentially important probes of the structure and physical conditions of the broad-line region (BLR) and of the unobservable ionizing continuum in these sources. Probably the best-established correlation between continuum and emission-line properties is the "Baldwin effect" (Baldwin 1977), an inverse, nonlinear correlation between the equivalent width of the broad UV emission lines, most notably C IV $\lambda 1549$, and the luminosity of the underlying continuum (e.g., Osmer, Porter, \& Green 1994). The Baldwin relationship can be parameterized as a simple power law of the form

$$
F_{\text {line }} \propto F_{\text {cont }}^{\alpha},
$$

with index $\alpha \lesssim 1$.

Kinney, Rivolo, \& Koratkar (1990) and Pogge \& Peterson (1992) investigated the effect of variability on this relationship in $\mathrm{C}$ IV and $\mathrm{Ly} \alpha$. The results can be summarized as follows:

1. There is a global or object-to-object relationship with slope $\alpha \approx 0.83$ for C IV and $\alpha \approx 0.88$ for $\operatorname{Ly} \alpha$.

2. There is a rather flatter intrinsic relationship with $\alpha \approx 0.4$ that describes how the line and continuum relationship changes because of variability. At least some of the scatter in the global relationship is attributable to variability and the flatter slope of the intrinsic relationship. Moreover, the scatter in this intrinsic relationship is largely attributable to light-travel time effects within the BLR (i.e., changes in the line flux follow those in the continuum flux with some measurable time delay).

While the Baldwin effect is well established in most of the strong UV lines, any global Baldwin effect in the Balmer lines seems to be quite weak (e.g., Dietrich et al. 2002). How-

\footnotetext{
${ }^{1}$ Current address: University of California Observatories, Lick Observatory, University of California, Santa Cruz, CA 95064.
}

ever, to the best of our knowledge, the question of whether or not there is an intrinsic relationship in the Balmer lines has not been specifically addressed. Our goal in this paper is to remedy this. We adopt an approach similar to that of Pogge \& Peterson (1992) in attempting to remove lighttravel time effects when determining the slope of the relationship. In this analysis we will make use of numerous well-sampled optical spectra of NGC 5548 obtained by the International AGN Watch to show that an intrinsic Baldwin effect is present in $\mathrm{H} \beta$.

\section{DATA ANALYSIS}

\subsection{The Data}

For this investigation, we used $10 \mathrm{yr}$ of spectra from the International AGN Watch collaboration (Peterson et al. 2002 and references therein). These data are fairly inhomogeneous, with differences in spectral resolution, quality, and amounts of contaminating light from the host galaxy. In order to minimize the effects of these problems, we restricted our analysis to the high-quality homogeneous data obtained with the Ohio State CCD spectrograph on the Perkins $1.8 \mathrm{~m}$ telescope at the Lowell Observatory in Flagstaff, Arizona, between 1988 and 1998. The optical continuum and $\mathrm{H} \beta$ emission-line fluxes for this subset are shown in Figure 1. The continuum flux was measured in a $10 \AA$ wide bin at a wavelength of $5100 \AA$ in the rest frame of the galaxy, which has a redshift of $z=0.017$. The continuum underneath the $\mathrm{H} \beta$ line was modeled by linear interpolation between two windows on either side of the $\mathrm{H} \beta$ line, at wavelengths of 4710 and $5100 \AA$ in the rest frame of the galaxy. The $\mathrm{H} \beta$ flux was then measured by integrating over the flux above this continuum. The measurement uncertainties in these spectral measurements were estimated by examining the differences in fluxes measured on very short timescales (i.e., less than or equal to 2 days). We assumed that flux differences on such short timescales are stochastic errors rather than real differences. We estimated the fractional error in the integrated continuum and line fluxes to be $2.5 \%$, consistent with 


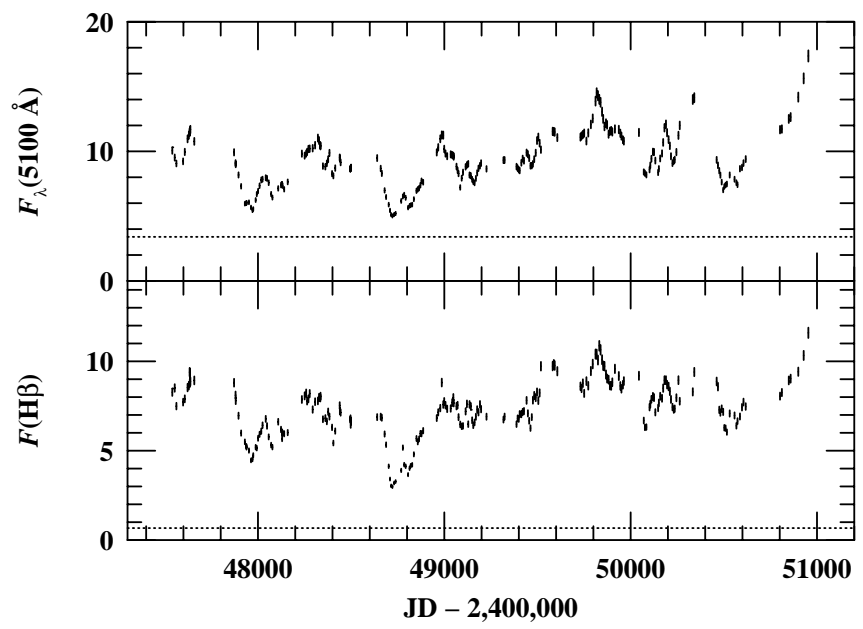

FIG. 1.-Optical continuum (upper panel) and $\mathrm{H} \beta$ (lower panel) light curves of NGC 5548 from 1989 December to 1998 June. The continuum fluxes are in units of $10^{-15} \mathrm{ergs} \mathrm{s}^{-1} \mathrm{~cm}^{-2} \AA^{-1}$, and the line fluxes are in units of $10^{-13} \mathrm{ergs} \mathrm{s}^{-1} \mathrm{~cm}^{-2}$. The dotted horizontal lines show the independent estimates of the constant components, specifically the continuum contribution from the host galaxy starlight (upper panel; Romanishin et al. 1995), and the narrow-line $\mathrm{H} \beta$ contribution (lower panel). Flux measurements are in the observer's reference frame and are uncorrected for Galactic extinction.

previous estimates of the error in these types of calculations (e.g., Peterson et al. 2002).

\subsection{Light-Travel Time Effects}

In this investigation it is important to take into account the effects of light-travel time. Continuum radiation emitted by the central continuum source (presumably an accretion disk) is absorbed and reprocessed into emission-line radiation by diffuse BLR gas. Because of light-travel time effects within the BLR, we observe a time delay of the order of days to weeks between variations in the continuum and the response of the emission lines. A simple linear model (Blandford \& McKee 1982) to describe the emission-line response to continuum variations can be written as

$$
L(t)=\int \Psi(\tau) C(t-\tau) d \tau,
$$

where $C(t)$ and $L(t)$ are the continuum and emission-line light curves, respectively, $\tau$ is the time delay, and $\Psi(\tau)$ is the transfer function. The transfer function depends on the size, geometry, and structure of the BLR. Under typical conditions, the centroid of the transfer function is essentially the mean response time of an emission line to changes in the continuum. The mean response time, or lag, for an emission line can be found by cross-correlation of the light curves.

In the case of NGC 5548, multiple years of monitoring optical continuum and emission-line variability has shown that the $\mathrm{H} \beta$ time lag changes over time. More precisely, the lag varies with the mean continuum level in a fashion that clearly suggests that the response is dominated by gas in which physical conditions are optimal for the $\mathrm{H} \beta$ line. Thus, the mean response time for a given line is a dynamic quantity and equation (2) is an overly simplistic model of the line response. However, it seems to be sufficient to account for time-dependent changes in the lag by taking yearly averages (Peterson et al. 2002).
We characterize the time delay by the value of the centroid of the optical continuum $/ \mathrm{H} \beta$ emission-line crosscorrelation functions for each year. The cross-correlation centroids are taken from Table 8 of Peterson et al. (2002); values range from a low of 11.37 days in 1992 to a high of 26.44 days in 1998. For each point in the $\mathrm{H} \beta$ light curve, a corresponding lag-corrected continuum point was determined. The lag was subtracted from the time of each $\mathrm{H} \beta$ measurement to identify the time at which the optical continuum was best correlated with the $\mathrm{H} \beta$ emission-line flux. The continuum flux at this time was determined by linear interpolation between the actual observations immediately preceding and following this time. Errors on interpolated continuum points were estimated by adding the errors of the preceding and following continuum points in quadrature, which probably slightly overestimates the actual uncertainty.

\subsection{Fitting Procedure and Results}

In Figure 2, we show the total $\mathrm{H} \beta$ flux as a function of optical continuum flux, corrected for time delay as described in the previous section. The relationship is clearly nonlinear. However, we have not yet accounted for constant spectral contaminants that have to be removed from the observed optical continuum and emission-line fluxes. The two major contaminants to the spectrum of importance to our investigation are $(a)$ the contribution of the host galaxy to the optical continuum and $(b)$ the $\mathrm{H} \beta$ narrow-line contribution to the total $\mathrm{H} \beta$ flux. Assuming that these are the only two necessary corrections, we can write equation (1) in terms of the observables, namely, the total continuum flux $F_{\lambda}(5100 \AA)$ (i.e., AGN plus host galaxy) and the total $\mathrm{H} \beta$ flux $F\left(\mathrm{H} \beta_{\text {total }}\right)$ (i.e., broad component plus narrow component) as

$$
\begin{aligned}
F\left(\mathrm{H} \beta_{\text {broad }}\right) & =F\left(\mathrm{H} \beta_{\text {total }}\right)-F\left(\mathrm{H} \beta_{\text {narrow }}\right) \\
& =A\left[F_{\lambda}(5100 \AA)-F_{\text {galaxy }}\right]^{\alpha} .
\end{aligned}
$$

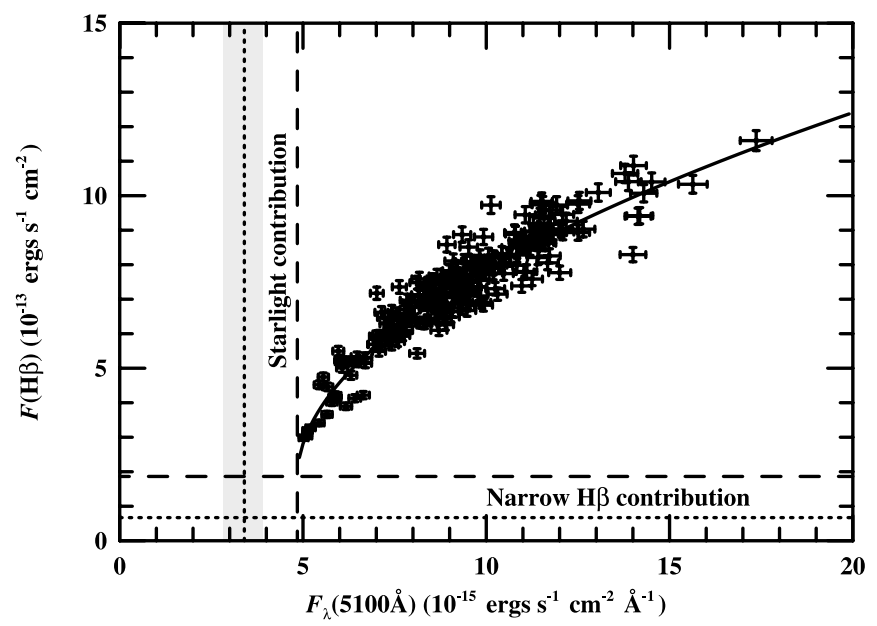

FIG. 2.-H $\beta$ vs. optical continuum flux for NGC 5548. The solid line shows the best fit to these data using a simple nonlinear form (eq. [3]). Dashed lines: Constant components (host galaxy flux and $\mathrm{H} \beta$ narrow-line contribution) based on this nonlinear fit. Dotted lines: Values of these quantities based on independent estimates, with the shaded area showing the $1 \sigma$ uncertainty in the host-galaxy flux. 
TABLE 1

Fits to the Optical ContinuUm-H $\beta$ Relationship

\begin{tabular}{ccccc}
\hline \hline \multicolumn{1}{c}{ Fit } & $\begin{array}{c}F\left(\mathrm{H} \beta_{\text {narrow }}\right) \\
\left(\times 10^{-13} \mathrm{ergs} \mathrm{s}^{-1} \mathrm{~cm}^{-2}\right)\end{array}$ & $\begin{array}{c}F_{\text {galaxy }} \\
\left(\times 10^{-15} \mathrm{ergs} \mathrm{s}^{-1} \mathrm{~cm}^{-2} \AA^{-1}\right)\end{array}$ & $A$ & $\alpha$ \\
\hline Nonlinear least-squares, eq. (3) .............. & $1.86 \pm 0.28$ & $4.85 \pm 0.08$ & $2.53 \pm 0.17$ & $0.526 \pm 0.022$ \\
Power-law fit, eq. (4) ............................ & $0.67^{\mathrm{a}}$ & $3.37^{\mathrm{a}}$ & $2.18 \pm 0.09$ & $0.648 \pm 0.022$ \\
\hline
\end{tabular}

a Parameter held fixed.

We can approach equation (3) in two ways, first by a complete nonlinear least-squares treatment, and second by fixing the host-galaxy and narrow-component fluxes, which are well constrained by independent analyses, which reduces the problem to a linear least-squares exercise. We consider both of these in turn.

Nonlinear least-squares fit.-We employed a Marquardt algorithm as described by Press et al. (1989) to determine the constant components $F\left(\mathrm{H} \beta_{\text {narrow }}\right)$ and $F_{\text {galaxy }}$, the scaling factor $A$, and the Baldwin effect index $\alpha$, all as defined in equation (3). The values of these parameters are shown in Table 1, along with uncertainties estimated by bootstrap resampling. The best-fit locus is shown in Figure 2.

Simple power-law fit.-We made use of the fact that two of the parameters in equation (3) can be accurately determined by independent means. The host-galaxy flux at 5100 $\AA$ is estimated to be $F_{\text {galaxy }}=(3.37 \pm 0.54) \times 10^{-15} \mathrm{ergs} \mathrm{s}^{-1}$ $\mathrm{cm}^{-2} \AA^{-1}$ (Romanishin et al. 1995) by using a model of the host-galaxy surface brightness distribution obtained by careful deconvolution of the nuclear point source and the host galaxy.

We used a value for the narrow $\mathrm{H} \beta$ line component of $F\left(\mathrm{H} \beta_{\text {narrow }}\right)=6.7 \times 10^{-14} \mathrm{ergs} \mathrm{s}^{-1} \mathrm{~cm}^{-2}$, which was estimated by trial-and-error scaling of the $[\mathrm{O}$ III] $\lambda 5007$ emission-line profile and subtracting the scaled profile at the position of the $\mathrm{H} \beta$ narrow line. This works best on highquality data obtained during a low-flux state, when the narrow component of $\mathrm{H} \beta$ is relatively prominent. A plausible narrow-line $\mathrm{H} \beta$ model was found by scaling the [O III] $\lambda 5007$ emission line by $0.12( \pm 0.01)$, as shown in Figure 3 .

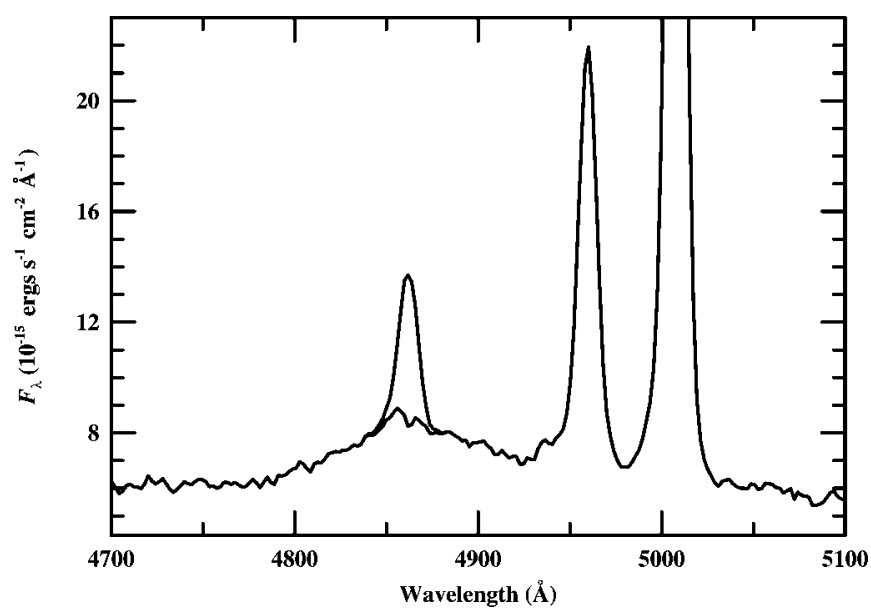

FIG. 3.- $\mathrm{H} \beta$ spectral region of NGC 5548 in a low state on JD 2,448, 726. The effect of attempting to remove the constant narrow-line component of $\mathrm{H} \beta$ is shown. The narrow-line $\mathrm{H} \beta$ component was removed by using the [O III] $\lambda 5007$ line as a template, scaled in flux by a factor of 0.12 .
After subtraction of these constant components, equation (3) reduces to a simple linear least-squares problem,

$\log F\left(\mathrm{H} \beta_{\text {broad }}\right)=\log A+\alpha \log \left[F_{\lambda}(5100 \AA)-F_{\text {galaxy }}\right]$.

The linear regression was performed using the BCES (bivariate correlated errors and intrinsic scatter) program described by Akritas \& Bershady (1996). This technique accounts for measurement errors in both parameters. The results for $\operatorname{BCES}(Y \| X)$ regression are given in Table 1, again with uncertainties in the fitted parameters determined by bootstrap resampling. The best-fit function is shown in Figure 4.

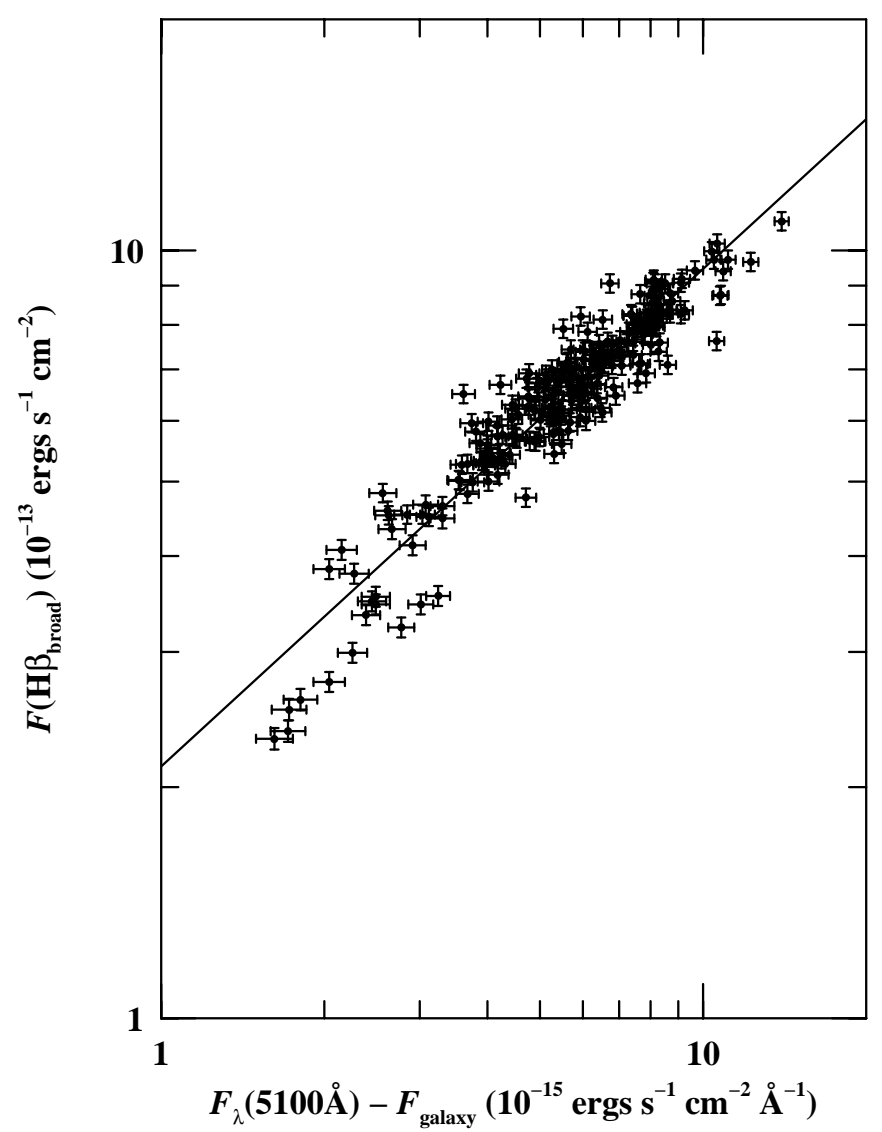

FIG. 4.-Broad $\mathrm{H} \beta$ flux as a function of optical nonstellar continuum flux for NGC 5548. The broad-line $\mathrm{H} \beta$ flux is obtained by subtracting an $\mathrm{H} \beta$ narrow-line contribution of $6.7 \times 10^{-14} \mathrm{ergs} \mathrm{s}^{-1} \mathrm{~cm}^{-2}$ from the total line measurement. The nonstellar continuum flux is obtained by subtracting a starlight contribution of $3.37 \times 10^{-15} \mathrm{ergs} \mathrm{s}^{-1} \mathrm{~cm}^{-2} \AA^{-1}$ from the optical continuum measurements. The solid line is the best fit of eq. (4) to these data, with slope $\alpha=0.648$ (Table 1). 


\section{DISCUSSION}

Both methods of fitting show a clear Baldwin effect, with $\alpha \approx 0.65$ for the simple linearized fit and $\alpha \approx 0.53$ for the nonlinear least-squares fit, in both cases rather steeper than the values found by Pogge \& Peterson (1992) for C IV and Ly $\alpha$. The fit shown in Figure 4 raises concern about either the parameterization of the relationship or the estimate of the background components (narrow-line $\mathrm{H} \beta$ and host galaxy); in other words, if the independent estimates of the background components are correct, then as shown in Figure 4 , most of the low-flux points fall below the best-fit line, indicating that the slope of the relationship must be different in this regime. While the nonlinear least-squares fit (Fig. 2) does not have this problem at the low-flux end, the background estimates from the fit are in both cases much too high, although certainly the constraint on the host-galaxy flux is less stringent than that on the $\mathrm{H} \beta$ narrow component. To pursue this, if we adjust upward from the Romanishin et al. (1995) estimate of the host-galaxy flux, we find the best overall fit (as measured by $\chi^{2}$ ) is achieved for a value of $4.6 \times 10^{-15} \mathrm{ergs} \mathrm{s}^{-1} \mathrm{~cm}^{-2} \AA^{-1}$ (yielding $\alpha \approx 0.52$ ), which is, not surprisingly, close to the host-galaxy estimate we obtained (Table 1) by the nonlinear least-squares fit to equation (3), the only difference being whether or not the narrow-line contribution is a free parameter or held fixed. A galaxy contribution of $4.6 \times 10^{-15}$ ergs $\mathrm{s}^{-1} \mathrm{~cm}^{-2} \AA^{-1}$ exceeds the Romanishin et al. (1995) estimate by $\sim 2 \sigma$, but it is still (although only slightly) below the minimum published optical continuum flux (Peterson et al. 2002). However, during the 2002 observing season, NGC 5548 went into the lowest flux state recorded over the last $30 \mathrm{yr}$, reaching a minimum $5100 \AA$ flux of $(3.94 \pm 0.10) \times 10^{-15} \mathrm{ergs} \mathrm{s}^{-1}$ $\mathrm{cm}^{-2} \AA^{-1}$ on JD $2,452,471$ (S. G. Sergeev 2002, private communication). Given this result, we doubt that the estimate of Romanishin et al. (1995) significantly underestimates the host-galaxy contribution; it seems more likely that our parameterization is oversimplified.

While Figure 4 suggests that the relationship between the continuum and the $\mathrm{H} \beta$ emission line changes at low flux levels, it is also possible that the relationship simply varies somewhat with time. As all of the low-flux points below the best-fit lines are from a single epoch (the fourth year of this monitoring program, during the period JD 2,448,713$2,448,848)$, we are unable to discriminate between flux dependence and time dependence. A complete analysis of the most recent faint-state data should clarify this.

Finally, as shown in Figure 3, our best estimate of the $\mathrm{H} \beta$ narrow component, based on a profile decomposition, is $6.7 \times 10^{-14} \mathrm{ergs} \mathrm{s}^{-1} \mathrm{~cm}^{-2}$. This is much lower than the nonlinear least-squares result of $1.8 \times 10^{-13} \mathrm{ergs} \mathrm{s}^{-1} \mathrm{~cm}^{-2}$ (Table 1; also see Fig. 2). It is very unlikely that the $\mathrm{H} \beta$ narrow component based on profile decomposition is underestimated. However, the nonlinear least-squares result may include other less variable contaminants to the broad $\mathrm{H} \beta$ profile, and this is under investigation.

Depending on how we account for the constant components, we find possible power-law indices (eq. [3]) in the range $0.52<\alpha<0.65$. In any case, we see clear evidence for an intrinsic Baldwin effect in $\mathrm{H} \beta$, i.e., the line flux does not increase linearly with the continuum flux. To explore the physical nature of this relationship, however, we need to relate the $\mathrm{H} \beta$ flux to the ionizing continuum flux rather than the optical continuum flux, since the former actually drives the emission-line variations. While the ionizing flux $(\lambda<912 \AA)$ is not directly observable, measurements closer to the ionizing continuum, at $1350 \AA$, are available from the International Ultraviolet Explorer archive. Assuming a relationship of the form

$$
F_{\text {opt }} \propto F_{\mathrm{UV}}^{\beta},
$$

Peterson et al. (2002) find $\beta \approx 0.56$, based on contemporaneous pairs of $1350 \AA\left(F_{\mathrm{UV}}\right)$ and $5100 \AA\left(F_{\text {opt }}\right)$ continuum fluxes. However, by using the BCES regression, we found a slightly steeper slope, $\beta=0.67$, which we will use in this discussion. We also note that in the case of NGC 5548, Galactic extinction is a small enough effect that it makes no difference in the slope $\beta$ of equation (5).

Our analysis thus far has been based only on optical data, and we have assumed specifically that $F(\mathrm{H} \beta) \propto F_{\mathrm{opt}}^{\alpha}$. Combining this with equation (5). we can write the relationship between the $\mathrm{H} \beta$ emission line flux and the UV ionizing continuum as $F(\mathrm{H} \beta) \propto F_{\mathrm{UV}}^{\alpha \beta}$, so that $0.35 \lesssim \alpha \beta \lesssim 0.44$.

\section{THE EFFECT OF GEOMETRIC DILUTION}

The emission-line light curve is a convolution of the continuum light curve with a transfer function, as shown in equation (2). The effect of the transfer function is to shift and broaden the emission-line light curve in time relative to the continuum curve; the effect of making the BLR larger is not only to shift the cross-correlation lag to larger values, but also to smear out the response in time, reducing the apparent amplitude of the response at any given time. We therefore consider here how important an effect this geometrical dilution of the line response might be in this particular case. We attempt to estimate this by convolving the real optical continuum light curve with a transfer function for a thin spherical shell, since this is a rectangular function that will have a relatively large dilution of the line response through time-smearing effects, and repeating the analysis described above.

The effect of geometric dilution on the amplitude of variability of emission lines was explored by convolving the NGC 5548 continuum with thin-shell transfer functions of radius between 1 and 10 lt-days. We then used the original continuum data and the simulated $\mathrm{H} \beta$ emission to calculate the slope of the Baldwin effect as discussed above. We found that the slope does decrease slightly with increasing geometric dilution; however, it does so at a very small rate. The difference in $\alpha$ is found to be only about 0.05 for rectangular transfer functions of half-width 1 and 10 days. We tested transfer functions for other geometries and found that, as expected, the effects of smearing were even smaller for narrower profile transfer functions.

\section{COMPARISON WITH THEORETICAL PREDICTIONS}

We can compare our empirical results on the intrinsic Baldwin effect in $\mathrm{H} \beta$ with the theoretical predictions made in a series of photoionization calculations by Korista et al. (1997; see their Fig. 3g). The quantity $\Phi(\mathrm{H})$ is defined as the number of ionizing photons arriving at the inner face of a BLR cloud per square centimeter per second. If the shape of the ionizing continuum is fixed, the emission-line spectrum will be a function of $\Phi(\mathrm{H})$, which is proportional to the photoionization rate, and of the particle density $n\left(\mathrm{~cm}^{-3}\right)$, which 
is proportional to the recombination rate, as well as chemical abundances and cloud column density. Korista et al. show the strength of various emission lines normalized by the continuum flux (i.e., the line equivalent widths) for various values of $\Phi(\mathrm{H})$ and $n$ for different column densities and standard assumptions about elemental abundances. For NGC $5548, \log \Phi(\mathrm{H})=20$ at a distance of 12.6 lt-days from the central source (Korista \& Goad 2001). The equivalent width used by Korista et al. is defined as the ratio of the $\mathrm{H} \beta$ flux to the continuum flux at $1215 \AA$. Using the above relationships, the equivalent width should be

$$
W=\frac{F(\mathrm{H} \beta)}{F_{\mathrm{UV}}} \propto F_{\mathrm{UV}}^{\alpha \beta-1} .
$$

Using our values of $\alpha$ and $\beta$, we find that we expect the equivalent width to be proportional to the UV flux to the -0.56 or -0.65 power, depending how we account for the constant components. In other words, an order-ofmagnitude change in $\Phi(\mathrm{H})$ should change the equivalent width by around -0.60 dex. From Figure $3 \mathrm{~g}$ of Korista et al. (1997), one can see that for $\log \Phi(\mathrm{H}) \approx 20$ and for hydrogen particle densities $n(\mathrm{H})\left(\mathrm{cm}^{-3}\right)$ in the range $11 \lesssim$ $\log n(\mathrm{H}) \lesssim 13$, the slope of the $\log W / \log \Phi(\mathrm{H})$ relationship is consistent with our results. The grids ${ }^{2}$ were investigated to find the slope of the relationship for varying values of column density and continuum shape with solar metallicity. Since the value of $\log \Phi(H) \approx 19.6$ at a radius of 20 lt-days,

\footnotetext{
${ }^{2}$ The photoionization grids calculated by Korista et al. (1997) can be
} found at http://www.pa.uky.edu/ korista/grids/grids.html. where typically the $\mathrm{H} \beta$ response is maximized in NGC 5548, we determined the slope of the grid for varying values of the hydrogen particle density by linear interpolation along the grid. We found that regardless of the model, the slope of the $\log W / \log \Phi(\mathrm{H})$ relationship reaches a maximum in the range of -0.57 to -0.60 , when the value of the hydrogen particle density is in the range $12 \lesssim \log n(\mathrm{H}) \lesssim 12.5$.

\section{CONCLUSIONS}

We have investigated the relationship between the variable $\mathrm{H} \beta$ emission line and optical continuum of NGC 5548. We discovered that an intrinsic Baldwin effect does exist; after correcting for light-travel time effects, we find that the amplitude of broad $\mathrm{H} \beta$ variation is less than the amplitude of variation of the underlying optical continuum. By combining these results with the relationship between the amplitudes of variability of the optical and UV continuum, we determine the relationship between the $\mathrm{H} \beta$ emission-line flux and the UV continuum flux and find that our result is in good agreement with the predictions of photoionization calculations, even accounting for smearing of the line response by light-travel time effects within the $\mathrm{H} \beta$-emitting region. We suggest that similar analysis of multiple lines have potential as diagnostics of the physical nature of the BLR.

We are grateful for support of this work through NASA grant NAG 5-8397. We thank Sergey Sergeev of the Crimean Astrophysical Observatory for data on the recent faint state of NGC 5548. We are grateful to an anonymous referee whose comments led to an improved presentation.
Akritas, M. G., \& Bershady, M. A. 1996, ApJ, 470, 706

Baldwin, J. 1977, ApJ, 214, 679

Blandford, R. D., \& McKee, C. F. 1982, ApJ, 255, 419

Dietrich, M., Hamann, F., Shields, J. C., Constantin, A., Vestergaard, M.,

Chaffee, F., Foltz, C. B., \& Junkkarinen, V. T. 2002, ApJ, 581, 912

Kinney, A. L., Rivolo, A. R., \& Koratkar, A. P. 1990, ApJ, 357, 338

Korista, K., Baldwin, J., Ferland, G., \& Verner, D. 1997, ApJS, 108, 401

\section{REFERENCES}

Korista, K. T., \& Goad, M. R. 2001, ApJ, 553, 695

Osmer, P. S., Porter, A. C., \& Green, R. F. 1994, ApJ, 436, 678

Peterson, B. M., et al. 2002, ApJ, 581, 197

Pogge, R. W., \& Peterson, B. M. 1992, AJ, 103, 1084

Press, W., Flannery, B., Teukolsky, S., \& Vetterling, W. 1989, in Numerical Recipes (Fortran) (Cambridge: Cambridge Univ. Press), 508

Romanishin, W., et al. 1995, ApJ, 455, 516 